\title{
INEQUALITIES FOR $\alpha$-OPTIMAL PARTITIONING OF A MEASURABLE SPACE
}

\author{
JERZY LEGUT
}

(Communicated by Daniel W. Strook)

\begin{abstract}
An $\alpha$-optimal partition $\left\{A_{i}^{*}\right\}_{i=1}^{n}$ of a measurable space according to $n$ nonatomic probability measures $\left\{\mu_{i}\right\}_{i=1}^{n}$ is defined. A two-sided inequality for $v^{*}=\min \alpha_{i}^{-1} \mu_{i}\left(A_{i}^{*}\right)$ is given. This estimation generalizes and improves a result of Elton et al. [3].
\end{abstract}

1. Introduction. Let $(\mathscr{X}, \mathscr{B})$ denote a measurable space and let $\left\{\mu_{i}\right\}_{i=1}^{n}$ be nonatomic probability measures defined on the same $\sigma$-algebra $\mathscr{B}$. Let $\mathscr{P}$ stand for the set of all measurable partitions $P=\left\{A_{i}\right\}_{i=1}^{n}$ of $\mathscr{X}\left(\bigcup_{i=1}^{n} A_{i}=\mathscr{X}, A_{i} \cap A_{j}=\varnothing\right.$, for all $i \neq j$ ).

Definition. A partition $P^{*}=\left\{A_{i}^{*}\right\}_{i=1}^{n} \in \mathscr{P}$ is said to be an $\alpha$-optimal if

$$
\min _{i \in I}\left\{\alpha_{i}^{-1} \mu_{i}\left(A_{i}^{*}\right)\right\}=\sup \left\{\min _{i \in I}\left\{\alpha_{i}^{-1} \mu_{i}\left(A_{i}\right)\right\}: P=\left\{A_{i}\right\}_{i=1}^{n} \in \mathscr{P}\right\}
$$

where $I=\{1,2, \ldots, n\}$ and $\alpha=\left(\alpha_{1}, \alpha_{2}, \ldots, \alpha_{n}\right) \in R^{n}$ is a vector satisfying $\sum_{i=1}^{n} \alpha_{i}=1$ and $\alpha_{i}>0$ for all $i \in I$.

The main purpose of this paper is to give as good as possible estimation of the number $v^{*}=\min _{i \in I}\left\{\alpha_{i}^{-1} \mu_{i}\left(A_{i}^{*}\right)\right\}$ by suitable inequalities. Using other simpler methods we generalize and improve the result of Elton et al. [3]. In $\S 2$ we state and prove the main theorem.

2. The main result. The problem of the $\alpha$-optimal partitioning of a measurable space $(\mathscr{X}, \mathscr{B})$ can be interpreted as the well-known problem of fair division (cf. $[\mathbf{1}, \mathbf{6}])$ of an object $\mathscr{X}$ with unequal weights. Here, each $\mu_{i}, i \in I$, represents the individual evaluation of sets from $\mathscr{B}$. We also assume in this problem that $\left\{\mu_{i}\right\}_{i=1}^{n}$ are nonatomic probability measures. Dividing the object $\mathscr{X}$ fairly we are interested in giving the $i$ th person a set $A_{i} \in \mathscr{B}$ such that $\mu_{i}\left(A_{i}\right) \geq \alpha_{i}$, for all $i \in I$.

Under assumptions given above, Dubins and Spanier [1] proved the following

THEOREM 1. Assume that $\mu_{i} \neq \mu_{j}$ for some $i \neq j$. Then there exists a partition $P=\left\{A_{i}\right\}_{i=1}^{n} \in \mathscr{P}$ such that $\mu_{i}\left(A_{i}\right)>\alpha_{i}$ for all $i \in I$.

The proof of Theorem 1 can be derived from the following result of Dvoretzky et al. [2] (cf. [1]).

Received by the editors June 15, 1987 and, in revised form, December 17, 1987.

1980 Mathematics Subject Classification (1985 Revision). Primary 60A10, 28A99; Secondary $60 \mathrm{E} 15,62 \mathrm{C} 20$. 

by

THEOREM 2. Let $\bar{\mu}: \mathscr{P} \rightarrow R^{n}$ denote the division vector valued function defined

$$
\bar{\mu}(P)=\left(\mu_{1}\left(A_{1}\right), \mu_{2}\left(A_{2}\right), \ldots, \mu_{n}\left(A_{n}\right)\right) \in R^{n}, \quad P=\left\{A_{i}\right\}_{i=1}^{n} \in \mathscr{P} .
$$

Then the range $\bar{\mu}(\mathscr{P})$ of $\bar{\mu}$ is convex and compact in $R^{n}$.

From Theorem 2 we conclude

COROLlaRY 1. There exists a partition $P^{*}=\left\{A_{i}^{*}\right\}_{i=1}^{n} \in \mathscr{P}$ such that

$$
v^{*}=\min _{i \in I}\left\{\alpha_{i}^{-1} \mu_{i}\left(A_{i}^{*}\right)\right\}=\sup \left\{\min _{i \in I}\left\{\alpha_{i}^{-1} \mu_{i}\left(A_{i}\right)\right\}: P=\left\{A_{i}\right\}_{i=1}^{n} \in \mathscr{P}\right\} .
$$

Thus the definition of the $\alpha$-optimal partition is correct.

A method of obtaining the $\alpha$-optimal partition was given by Legut and Wilczyński [5].

COROLlaRY 2. There exists a partition $P^{0}=\left\{A_{i}^{0}\right\}_{i=1}^{n} \in \mathscr{P}$ such that

$$
M:=\sum_{i=1}^{n} \mu_{i}\left(A_{i}^{0}\right)=\sup \left\{\sum_{i=1}^{n} \mu_{i}\left(A_{i}\right): P=\left\{A_{i}\right\}_{i=1}^{n} \in \mathscr{P}\right\} .
$$

Denote $p_{i}:=\mu_{i}\left(A_{i}^{0}\right), p:=\left(p_{1}, p_{2}, \ldots, p_{n}\right)$ and

$$
m:=\min \left\{p_{i}\left[p_{i}-\alpha_{i}(M-1)\right]^{-1}:\left[p_{i}-\alpha_{i}(M-1)\right]>0, i \in I\right\} .
$$

The number $M$ can be interpreted as the "cooperative" value of the fair division problem (cf. [4]). It is clear that if $\mu_{i} \neq \mu_{j}$ for some $i \neq j$ then $M>1$.

Now we can state our main result.

THEOREM 3. $m \leq v^{*} \leq M$.

PrOOF. At first we show the inequality $v^{*} \leq M$. Suppose that $v^{*}>M$. From the definition of the number $v^{*}$ we obtain $\alpha_{i}^{-1} \mu_{i}\left(A_{i}^{*}\right)>M$, for all $i \in I$. Hence we have $\sum_{i=1}^{n} \mu_{i}\left(A_{i}^{*}\right)>M$. This inequality contradicts the definition of the number $M$.

To prove that $m \leq v^{*}$ we put $e_{i}=(0, \ldots, 1, \ldots, 0) \in R^{n}$ (1 is placed on the $i$ th coordinate, $i \in I)$. Clearly, $e_{i} \in \bar{\mu}(\mathscr{P})$, for all $i \in I$. Let $V$ denote the convex hull of the set $\left\{p,\left\{e_{i}\right\}_{i=1}^{n}\right\}$. From Theorem 2 we have $V \subset \bar{\mu}(\mathscr{P})$. It is now sufficient to find a real number $t^{*}:=\max \{t \in R: t \alpha \in V\}$. Solving the following system of $n+1$ linear equalities

$$
\begin{aligned}
& \beta_{i}+\beta_{n+1} p_{i}=\alpha_{i} t, \quad i \in I, \\
& \sum_{i=1}^{n} \beta_{i}=1,
\end{aligned}
$$

with respect to $\beta_{i} \geq 0, i=1,2, \ldots, n+1$, we obtain $t^{*}=m$. Hence we conclude that $m \leq v^{*}$ and the proof is complete.

REMARK. Let $\alpha_{i}=n^{-1}$ for all $i \in I$. In this case we get from Theorem 3

$$
p^{*}\left[p^{*}-n^{-1}(M-1)\right]^{-1} \leq v^{*} \leq M
$$

where $p^{*}:=\max \left\{p_{i}: i \in I\right\}$. Since $p^{*} \leq 1$ we obtain

$$
n[n-(M-1)]^{-1} \leq n p^{*}\left[n p^{*}-(M-1)\right]^{-1} \text {. }
$$


Finally, we have the result of Elton et al. [3]

$$
[n-(M-1)]^{-1} \leq n^{-1} v^{*} \leq n^{-1} M .
$$

EXAMPLE. Let $\mathscr{X}=[0,1]$ and $\mathscr{B}$ be the $\sigma$-algebra of Borel subsets of $[0,1]$. Let $\lambda$ be the Lebesgue measure on $[0,1]$. We consider the case $n=2$ with $\alpha_{1}=\alpha_{2}=$ $1 / 2$. Define $\mu_{1}=\lambda$ and $\mu_{2}(A)=2 \lambda(A \cap[0,1 / 4))+(2 / 3) \lambda(A \cap(1 / 4,1])$ for $A \in \mathscr{B}$. It is easy to verify that $p_{1}=\mu_{1}((1 / 4,1])=3 / 4, p_{2}=\mu_{2}([0,1 / 4))=1 / 2$ and $M=p_{1}+p_{2}=5 / 4$. The result of Elton et al. [3] gives the following inequalities for the number $v^{*}$ :

$$
8 / 7 \leq v^{*} \leq 5 / 4
$$

but using the estimation from Theorem 3 we obtain

$$
8 / 7<6 / 5 \leq v^{*} \leq 5 / 4 \text {. }
$$

\section{REFERENCES}

1. L. Dubins and E. Spanier, How to cut a cake fairly, Amer. Math. Monthly 68 (1961), 1-17.

2. A. Dvoretzky, A. Wald and J. Wolfowitz, Relations among certain ranges of vector measures, Pacific J. Math. 1 (1951), 59-74.

3. J. Elton, T. Hill and R. Kertz, Optimal partitioning inequalities for nonatomic probability measures, Trans. Amer. Math. Soc. 296 (1986), 703-725.

4. J. Legut, Market games with continuum of indivisible commodities, J. Game Theory 15 (1986), 1-7.

5. J. Legut and M. Wilczyński, Optimal partitioning of a measurable space, Proc. Amer. Math. Soc. Volume 104, Number 1, September 1988.

6. H. Steinhaus, Sur la division pragmatique, Econometrica Supplement 17 (1949), 315-319.

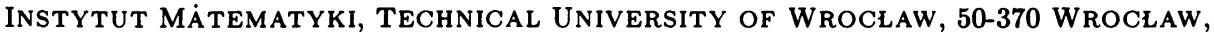
POLAND 